\title{
Existence Solution for Nonlinear System of Fractional Integrodifferential Equations of Volterra Type with Fractional Boundary Conditions
}

\author{
Faraj Y. Ishak \\ University of Duhok Iraq, faraj.ishak@uod.ac \\ doi: https://doi.org/10.15642/mantik.2020.6.1.1-12
}

\begin{abstract}
Abstrak: Artikel ini menyelidiki eksistensi, keunikan, dan solusi stabil dari sistem persamaan diferensial-diferensial Volterra fraksional baru dengan kondisi batas fraksional dengan menggunakan teorema eksistensi dan keunikan. Teorema tentang eksistensi dan keunikan dari solusi yang ditetapkan di bawah beberapa kondisi yang diperlukan dan cukup pada ruang kompak. Contoh sederhana dari hasil aplikasi utama disajikan dalam artikel ini.
\end{abstract}

Kata kunci: Keberadaan dan keunikan, stabilitas, fractional Integrodifferential equations, masalah nilai batas, teorema eksistensi dan keunikan.

Abstract: This article investigates existence, uniqueness and stability solutions of new fractional Volterra integro-differential equations system with fractional boundary conditions by using the existence and uniqueness theorem. Theorems on existence and uniqueness of solution are established under some necessary and sufficient conditions on compact space. A simple example of application of the main results of this article is presented.

Keywords: Existence and uniqueness, stability, fractional Integrodifferential equations, boundary value problem, existence and uniqueness theorem. 


\section{Introduction}

Gottfried Leibniz and Guilliaume L'Hopital sparked initial curiosity into the theory of fractional calculus during a 1695 correspondence on the possible value and meaning of non-integer-order derivatives. By the late nineteenth century, the combined efforts of a number of mathematicians most notably Liouville, Grunwald, Letnikov, and Riemann produced a fairly solid theory of fractional calculus for functions of a real variable. Though several viable fractional derivatives were proposed, the so-called RiemannLiouville and Caputo derivatives are the two most-commonly used today.

Mathematicians have employed this fractional calculus in recent years to model and solve a variety of applied problems. Indeed, as Podlubney outlines in [1].

Fractional differential equations have extensive applications in various fields of science and engineering. Many phenomena in viscoelasticity, electrochemistry, control theory, porous media, electromagnetism, and other fields, can be modelled by fractional differential equations. We refer the reader to $[2,4]$ and references therein for some applications. Fractional BVPs defined on intervals have been studied by many authors. Many results on the existence, uniqueness, multiplicity, and nonexistence of solutions for fractional differential equations subject to various boundary conditions (BCs) have been obtained; see for example $[9,10,11,12,13,14,15,16]$.

The fractional difference calculus had its origin in the works by Al-Salam [6] and Agarwal [7]. More recently, perhaps due to the explosion in research within the fractional differential calculus setting, new developments in this theory of fractional difference calculus were made, specifically, analogues of the integral and differential fractional operators properties such as the Mittag-Leffler function, the Laplace transform, and Taylor's formula $[3,5,8,17,18]$, just to mention some.

Butris and Ishak [20], used both methods Picard approximation and Banach fixed point theorems for studying the existence and uniqueness solutions to the following fractional integral equations:

$$
\begin{aligned}
u(t) & =f(t)+\frac{1}{\Gamma(\alpha)} \int_{a}^{t}(t-s)^{\alpha-1} F(t, s, u(s), w(s)) d s \\
w(t) & =g(t)+\frac{1}{\Gamma(\alpha)} \int_{a}^{b}(t-s)^{\alpha-1} G(t, s, u(s), w(s)) d s
\end{aligned}
$$

In this work our aim is to show the existence solutions of the system of integrodifferential equations

Where:

$$
\begin{gathered}
D^{\alpha} x(t)+f(t, s,[\phi x](x))=0 \\
D^{\beta} y(t)+g(t, s,[\varphi y](y))=0 \\
D^{\alpha-1} x(0)=0, \quad D^{\alpha-1} x(1)=b_{1}, \\
D^{\beta-1} y(0)=0, \quad D^{\beta-1} y(1)=b_{2}
\end{gathered}
$$

$[\phi x](x)=\int_{-\infty}^{t} K(t, s) F(t, s, x(s), y(s)) d s, \quad[\varphi y](y)=$ $\int_{-\infty}^{t} G(t, s) H(t, s, x(s), y(s)) d s$

$1<\alpha, \beta \leq 2,0 \leq s \leq t \leq 1, b_{1}, b_{2} \in R, x \in D_{1} \subseteq R^{n}$ and $y \in D_{2} \subseteq R^{m}, D_{1}$ and $D_{2}$ are compact domain, let the vector functions $F(t, s, x(t), y(t)), H(t, s, x(t), y(t))$ is defined and continuous on the domain:

$$
D=\left\{(t, s, x, y) ; t, s \in[0,1], x \in D_{1}, y \in D_{2}\right\}
$$


F. Y. Ishak

Assume that the vector functions $F(t, s, x(t), y(t)), H(t, s, x(t), y(t))$, and kernels $K(t, s), G(t, s)$ are satisfying the following inequalities:

$$
\begin{aligned}
& \|F(t, s, x(t), y(t))\| \leq M_{1},\|H(t, s, x(t), y(t))\| \leq M_{2} \\
& \left\|F\left(t, s, x_{2}, y_{2}\right)-F\left(t, s, x_{1}, y_{1}\right)\right\| \leq L_{1}\left(\left\|x_{2}-x_{1}\right\|+\left\|y_{2}-y_{1}\right\|\right) \\
& \left\|H\left(t, s, x_{2}, y_{2}\right)-H\left(t, s, x_{1}, y_{1}\right)\right\| \leq L_{2}\left(\left\|x_{2}-x_{1}\right\|+\left\|y_{2}-y_{1}\right\|\right) \\
& \|K(t, s)\| \leq \delta_{1} e^{-\lambda_{1}(t-s)}, \quad\|G(t, s)\| \leq \delta_{2} e^{-\lambda_{2}(t-s)}
\end{aligned}
$$

Where $M_{1}, M_{2}, L_{1}, L_{2}, \lambda_{1}, \lambda_{2}, \delta_{1}, \delta_{2}$, are positive constants $x_{1}, x_{2} \in D_{1} y_{1}, y_{2} \in D_{2}$ $t, s \in[0,1]$ and $\|\|=.\max _{t \in[0,1]} \mid$. |, we defined non-empty sets as:

$$
\left.\begin{array}{l}
D_{F}=D_{1}-\frac{M_{1} \delta_{1}(\alpha-1)+b_{1} \lambda_{1} \alpha}{\lambda_{1} \Gamma(\alpha+1)} \\
D_{H}=D_{2}-\frac{M_{2} \delta_{2}(\beta-1)+\lambda_{2} b_{2} \beta}{\lambda_{2} \Gamma(\beta+1)}
\end{array}\right\}
$$

As well as we suppose the maximum value of the following matrix:

$$
\begin{aligned}
& \Delta_{0}=\left(\begin{array}{ll}
\frac{L_{1} \delta_{1}(\alpha-1)}{\lambda_{1} \Gamma(\alpha+1)} & \frac{L_{1} \delta_{1}(\alpha-1)}{\lambda_{1} \Gamma(\alpha+1)} \\
\frac{L_{2} \delta_{2}(\beta-1)}{\lambda_{2} \Gamma(\beta+1)} & \frac{L_{2} \delta_{2}(\beta-1)}{\lambda_{2} \Gamma(\beta+1)}
\end{array}\right), \text { less than one i.e. } \\
& \lambda_{\text {max }}\left(\Delta_{0}\right)=\frac{L_{1} \delta_{1}(\alpha-1)}{\lambda_{1} \Gamma(\alpha+1)}+\frac{L_{2} \delta_{2}(\beta-1)}{\lambda_{2} \Gamma(\beta+1)}<1
\end{aligned}
$$

Define a sequence of functions $\left\{x_{m}\left(t, x_{0}\right)\right\}_{m=0}^{\infty},\left\{y_{m}\left(t, y_{0}\right)\right\}_{m=0}^{\infty}$ as:

$$
\left.\begin{array}{c}
x_{m+1}\left(t, x_{0}\right)=\frac{t^{\alpha-1}}{\Gamma(\alpha)} \int_{0}^{1} \int_{-\infty}^{t} K(t, s) F\left(t, s, x_{m}(s), y_{m}(s)\right) d s d s- \\
\int_{0}^{t(t-s)^{\alpha-1}} \frac{\left(\int_{-\infty}^{t} K(t, s) F\left(t, s, x_{m}(s), y_{m}(s)\right) d s\right) d s+\frac{b_{1} t^{\alpha-1}}{\Gamma(\alpha)}}{\Gamma(\alpha)} \\
y_{m+1}\left(t, y_{0}\right)=\frac{t^{\beta-1}}{\Gamma(\beta)} \int_{0}^{1} \int_{-\infty}^{t} G(t, s) H\left(t, s, x_{m}(s), y_{m}(s)\right) d s d s- \\
\int_{0}^{t} \frac{(t-s)^{\beta-1}}{\Gamma(\beta)}\left(\int_{-\infty}^{t} G(t, s) H\left(t, s, x_{m}(s), y_{m}(s)\right) d s\right) d s+\frac{b_{2} t^{\beta-1}}{\Gamma(\beta)}
\end{array}\right\}
$$

With $x_{0}=x(0)=0, \quad y_{0}=y(0)=0, \quad \mathrm{~m}=0,1,2 \ldots$

\section{Preliminaries}

Let us recall some basic definitions on fractional calculus, which can be found in the literature.

Definition 2.1 [19] Assume that $f(x, y)$ is defined on the set $(a, b) \mathrm{x} G, G \subset R, f(x, y)$ is said to satisfy Lipschitz condition with respect to the second variable, if for all $x \in$ $(a, b)$ and for any $y_{1}, y_{2} \in G$

$$
\left|f\left(x, y_{1}\right)-f\left(x, y_{2}\right)\right| \leq \xi\left|y_{1}-y_{2}\right|
$$


where $\xi>0$ does not depend on $x \in(a, b)$.

Definition 2.2 [19] The Riemann-Liouville fractional integral of order q is defined by

provided the integral exists.

$$
I^{q} f(t)=\frac{1}{\Gamma(q)} \int_{0}^{t}(t-s)^{q-1} f(s) d s, \quad q>0
$$

Definition 2.3 [19] The Riemann-Liouville fractional derivative of order q is defined by

$$
D^{q} f(t)=\frac{1}{\Gamma(n-q)}\left(\frac{d}{d t}\right)^{n} \int_{0}^{t}(t-s)^{n-q-1} f(s) d s, \quad n-1<q \leq n, \quad q>0,
$$

Provided the right-hand side is pointwise defined on $(0,+\infty)$.

Lemma 2.1 For $\alpha, \beta>0$, then the following relation hold:

$$
D^{\alpha} t^{\beta}=\frac{\Gamma(\beta+1)}{\Gamma(\beta+1-\alpha)} t^{\beta-\alpha-1}, \beta>n \text { and } D^{\alpha} t^{k}=0, k=0,1, \ldots, n-1
$$

Lemma 2.2 [3] The equality $D_{0+}^{\alpha} I_{0+}^{\alpha} f(t)=f(t), \quad \alpha>0$ holds for $f \in L^{1}(0,1)$.

Lemma 2.3 Let $\alpha, \beta>0$ and let $\mathrm{f}$ be a function defined on $[0,1]$. Then the following formulas hold:

$$
\begin{array}{ll}
\text { (i) } & \left(I_{q}^{\beta} I_{q}^{\alpha} f\right)(x)=\left(I_{q}^{\alpha+\beta} f\right)(x) \\
\text { (ii) } & \left(D_{q}^{\alpha} I_{q}^{\alpha} f\right)(x)=f(x)
\end{array}
$$

Lemma 2.4 Let $\alpha>0$ and $n$ be a positive integer. Then, the following equality holds:

$$
\left(I_{q}^{\beta} D_{q}^{\alpha} f\right)(x)=\left(D_{q}^{n} I_{q}^{\alpha} f\right)(x)-\sum_{k=0}^{n-1} \frac{x^{\alpha-n+k}}{\Gamma(\alpha+\mathrm{k}-\mathrm{n}+1)}\left(D_{q}^{k} f\right)(0)
$$

Lemma 2.5 A functions $x(t), y(t)$, are solution of system (1.1) if and only if $x(t), y(t)$ have the form:

$$
\begin{aligned}
x(t)=\frac{t^{\alpha-1}}{\Gamma(\alpha)} \int_{0}^{1} & \int_{-\infty}^{t} K(t, s) F(t, s, x(s), y(s)) d s d s \\
& -\int_{0}^{t} \frac{(t-s)^{\alpha-1}}{\Gamma(\alpha)}\left(\int_{-\infty}^{t} K(t, s) F(t, s, x(s), y(s)) d s\right) d s+\frac{b_{1} t^{\alpha-1}}{\Gamma(\alpha)} \\
y(t)=\frac{t^{\beta-1}}{\Gamma(\beta)} \int_{0}^{1} \int_{-\infty}^{t} G(t, s) H(t, s, x(s), y(s)) d s d s & -\int_{0}^{t} \frac{(t-s)^{\beta-1}}{\Gamma(\beta)}\left(\int_{-\infty}^{t} G(t, s) H\left(t, s, x(s), y_{m}(s)\right) d s\right) d s+\frac{b_{2} t^{\beta-1}}{\Gamma(\beta)}
\end{aligned}
$$

Proof: It follows from Lemma 2.4 that the system of fractional differential equation in (1.1) is equivalent to the integral equations:

$$
\begin{gathered}
x(t)=-I^{\alpha} f(t)+c_{1} t^{\alpha-1}+c_{2} t^{\alpha-2} \\
y(t)=-I^{\alpha} g(t)+d_{1} t^{\alpha-1}+d_{2} t^{\alpha-2}
\end{gathered}
$$


Where:

$f(t)=\int_{-\infty}^{t} K(t, s) F(t, s, x(s), y(s)) d s, g(t)=\int_{-\infty}^{t} G(t, s) H(t, s, x(s), y(s)) d s$, $c_{1}, c_{2}, d_{1}, d_{2} \in R$

From the boundary conditions of (1.1) we have $c_{2}=0, d_{2}=0$ and:

$$
c_{1}=\frac{b_{1}}{\Gamma(\alpha)}+\frac{1}{\Gamma(\alpha)} \int_{0}^{1} f(s) d s, d_{1}=\frac{b_{2}}{\Gamma(\beta)}+\frac{1}{\Gamma(\beta)} \int_{0}^{1} g(s) d s
$$

Which is complete the proof.

\section{Result and Discussion}

In this section, the theorems of existence, uniqueness, and stability of a solution for system (1.1) will be given.

Theorem 3.1: Let the right side of system (1.1) are defined and continuous on domain (1.2) .Suppose that the vector functions $F(t, s, x(t), y(t)), H(t, s, x(t), y(t))$ are satisfying the inequalities (1.3)-(1.5) and the conditions (1.6)-(1.9).Then there exist a sequences of functions (1.9) converges uniformly as $m \rightarrow \infty$ on domain (1.2) to the limit functions which satisfying integral equations (2.1) Provided that:

$$
\begin{aligned}
& \left\|x_{\infty}\left(t, x_{0}\right)-x_{0}\right\| \leq \frac{M_{1} \delta_{1}(\alpha-1)+\lambda_{1} b_{1} \alpha}{\lambda_{1} \Gamma(\alpha+1)} \\
& \left\|y_{\infty}\left(t, x_{0}\right)-y_{0}\right\| \leq \frac{M_{2} \delta_{2}(\beta-1)+\lambda_{2} b_{2} \beta}{\lambda_{2} \Gamma(\beta+1)} \\
& \} \\
& \left(\begin{array}{l}
\left\|x_{m+1}\left(t, x_{0}\right)-x_{m}\left(t, x_{0}\right)\right\| \\
\left\|y_{\mathrm{m}+1}\left(t, y_{0}\right)-y_{\mathrm{m}}\left(t, y_{0}\right)\right\|
\end{array}\right) \leq \Delta_{0}^{m}\left(I-\Delta_{0}\right)^{-1} \Phi_{0} \text { for all } m \geq 1, t \in[0,1]
\end{aligned}
$$

Proof:

By using the sequence of function (1.9) when $\mathrm{m}=0$, we get:

$$
\begin{aligned}
& \left\|x_{1}\left(t, x_{0}\right)-x_{0}\right\| \leq \frac{t^{\alpha-1}}{\Gamma(\alpha)} \int_{0}^{1} \int_{-\infty}^{t}\|K(t, s)\|\left\|F\left(t, s, x_{0}(s), y_{0}(s)\right)\right\| d s d s- \\
& \int_{0}^{t(t-s)^{\alpha-1}} \frac{\int_{-\infty}^{t}}{\Gamma(\alpha)}\left(\int_{-\infty}^{t}\|K(t, s)\|\left\|F\left(t, s, x_{0}(s), y_{0}(s)\right)\right\| d s\right) d s+\frac{b_{1} t^{\alpha-1}}{\Gamma(\alpha)} \\
& \leq \frac{t^{\alpha-1} M_{1}}{\Gamma(\alpha)} \int_{0}^{1} \int_{-\infty}^{t} \delta_{1} e^{-\lambda_{1}(t-s)} d s d s-\int_{0}^{t} \frac{(t-s)^{\alpha-1}}{\Gamma(\alpha)}\left(\int_{-\infty}^{t} \delta_{1} e^{-\lambda_{1}(t-s)} d s\right) d s+\frac{b_{1} t^{\alpha-1}}{\Gamma(\alpha)} \\
& \leq \frac{M_{1} \delta_{1}}{\lambda_{1} \Gamma(\alpha)}-\frac{M_{1} \delta_{1}}{\lambda_{1} \Gamma(\alpha+1)}+\frac{b_{1}}{\Gamma(\alpha)} \leq \frac{M_{1} \delta_{1}(\alpha-1)+b_{1} \lambda_{1} \alpha}{\lambda_{1} \Gamma(\alpha+1)}
\end{aligned}
$$

And by the same we have

$$
\left\|y_{1}\left(t, y_{0}\right)-y_{0}\right\| \leq \frac{M_{2} \delta_{2}(\beta-1)+\lambda_{2} b_{2} \beta}{\lambda_{2} \Gamma(\beta+1)}
$$

That is: $\quad x_{1}\left(t, x_{0}\right) \epsilon D_{1} y_{1}\left(t, y_{0}\right) \epsilon D_{2}$, for all $t \epsilon[0,1], x_{0} \in D_{F}, y_{0} \in D_{H}$ 
Suppose that $x_{p}\left(t, x_{0}\right) \in D_{1} \quad, y_{p}\left(t, y_{0}\right) \in D_{2}$ for each $x_{0} \in D_{F}, y_{0} \in D_{H} \quad, p \in Z^{+}, \mathrm{t} \in$ $[0,1]$,by mathematical induction we conclude that: $x_{m}\left(t, x_{0}\right) \in D_{1}, y_{m}\left(t, y_{0}\right) \in D_{2}$ for each $x_{0} \in D_{F}, y_{0} \in D_{H} \quad, m=0,1,2, \ldots$

To prove that the sequences (1.9) convergence uniformly in domain (1.2):

$$
\begin{aligned}
& \left\|x_{2}\left(t, x_{0}\right)-x_{1}\left(t, x_{0}\right)\right\| \leq \frac{t^{\alpha-1}}{\Gamma(\alpha)} \int_{0}^{1} \int_{-\infty}^{t}\|K(t, s)\|\left\|F\left(t, s, x_{1}(s), y_{1}(s)\right)\right\| d s d s- \\
& \int_{0}^{t} \frac{(t-s)^{\alpha-1}}{\Gamma(\alpha)}\left(\int_{-\infty}^{t}\|K(t, s)\|\left\|F\left(t, s, x_{1}(s), y_{1}(s)\right)\right\| d s\right) d s- \\
& \frac{t^{\alpha-1}}{\Gamma(\alpha)} \int_{0}^{1} \int_{-\infty}^{t}\|K(t, s)\|\left\|F\left(t, s, x_{0}(s), y_{0}(s)\right)\right\| d s d s+ \\
& \int_{0}^{t} \frac{(t-s)^{\alpha-1}}{\Gamma(\alpha)}\left(\int_{-\infty}^{t}\|K(t, s)\|\left\|F\left(t, s, x_{0}(s), y_{0}(s)\right)\right\| d s\right) d s \\
& \leq \frac{t^{\alpha-1}}{\Gamma(\alpha)} \int_{0}^{1} \int_{-\infty}^{t} \delta_{1} e^{-\lambda_{1}(t-s)}\left\|F\left(t, s, x_{1}(s), y_{1}(s)\right)-F\left(t, s, x_{0}(s), y_{0}(s)\right)\right\| d s d s \\
& -\int_{0}^{t} \frac{(t-s)^{\alpha-1}}{\Gamma(\alpha)}\left(\int_{-\infty}^{t} \delta_{1} e^{-\lambda_{1}(t-s)}\left\|F\left(t, s, x_{1}(s), y_{1}(s)\right)-F\left(t, s, x_{0}(s), y_{0}(s)\right)\right\| d s\right) d s \\
& \leq \frac{L_{1} t^{\alpha-1}}{\Gamma(\alpha)}\left(\left\|x_{1}-x_{0}\right\|+\left\|y_{1}-y_{0}\right\|\right) \int_{0}^{1} \int_{-\infty}^{t} \delta_{1} e^{-\lambda_{1}(t-s)} d s d s- \\
& L_{1}\left(\left\|x_{1}-x_{0}\right\|+\left\|y_{1}-y_{0}\right\|\right) \int_{0}^{t} \frac{(t-s)^{\alpha-1}}{\Gamma(\alpha)}\left(\int_{-\infty}^{t} \delta_{1} e^{-\lambda_{1}(t-s)} d s\right) d s \\
& \leq \frac{L_{1} \delta_{1}\left(\alpha t^{\alpha-1}-t^{\alpha}\right)}{\lambda_{1} \Gamma(\alpha+1)}\left(\left\|x_{1}-x_{0}\right\|+\left\|y_{1}-y_{0}\right\|\right)
\end{aligned}
$$

And by the same

$$
\left\|y_{2}\left(t, y_{0}\right)-y_{1}\left(t, y_{0}\right)\right\| \leq \frac{L_{2} \delta_{2}\left(\beta t^{\beta-1}-t^{\beta}\right)}{\lambda_{2} \Gamma(\beta+1)}-\left(\left\|x_{1}-x_{0}\right\|+\left\|y_{1}-y_{0}\right\|\right)
$$

By the mathematical induction the following inequalities hold:

$$
\begin{aligned}
& \left\|x_{m+1}\left(t, x_{0}\right)-x_{m}\left(t, x_{0}\right)\right\| \leq \frac{L_{1} \delta_{1}\left(\alpha t^{\alpha-1}-t^{\alpha}\right)}{\lambda_{1} \Gamma(\alpha+1)}\left(\left\|x_{m}-x_{m-1}\right\|+\left\|y_{m}-y_{m} \jmath_{1}\right\|\right) \\
& \left\|y_{m+1}\left(t, y_{0}\right)-y_{m}\left(t, y_{0}\right)\right\| \leq \frac{L_{2} \delta_{2}\left(\beta t^{\beta-1}-t^{\beta}\right)}{\lambda_{2} \Gamma(\beta+1)}\left(\left\|x_{m}-x_{m-1}\right\|+\left\|y_{m}-y_{m-1}\right\|\right)
\end{aligned}
$$

Rewrite (3.2) with vector form:

$$
\left(\begin{array}{ll}
\left\|x_{m+1}\left(t, x_{0}\right)-x_{m}\left(t, x_{0}\right)\right\| \\
\left\|y_{\mathrm{m}+1}\left(t, y_{0}\right)-y_{\mathrm{m}}\left(t, y_{0}\right)\right\|
\end{array}\right) \leq\left(\begin{array}{ll}
\frac{L_{1} \delta_{1}\left(\alpha t^{\alpha-1}-t^{\alpha}\right)}{\lambda_{1} \Gamma(\alpha+1)} & \frac{L_{1} \delta_{1}\left(\alpha t^{\alpha-1}-t^{\alpha}\right)}{\lambda_{1} \Gamma(\alpha+1)} \\
\frac{L_{2} \delta_{2}\left(\beta t^{\beta-1}-t^{\beta}\right)}{\lambda_{2} \Gamma(\beta+1)} & \frac{L_{2} \delta_{2}\left(\beta t^{\beta-1}-t^{\beta}\right)}{\lambda_{2} \Gamma(\beta+1)}
\end{array}\right)\left(\begin{array}{ll}
\left\|x_{m}-x_{m-1}\right\| \\
\left\|y_{m}-y_{m-1}\right\|
\end{array}\right)
$$

That is:

$$
\Phi_{m+1}\left(t, x_{0}, y_{0}\right) \leq \Delta(t) \Phi_{m}\left(t, x_{0}, y_{0}\right)
$$

Where: 
F. Y. Ishak

$$
\begin{aligned}
& \Delta(t)=\left(\begin{array}{ll}
\frac{L_{1} \delta_{1}\left(\alpha t^{\alpha-1}-t^{\alpha}\right)}{\lambda_{1} \Gamma(\alpha+1)} & \frac{L_{1} \delta_{1}\left(\alpha t^{\alpha-1}-t^{\alpha}\right)}{\lambda_{1} \Gamma(\alpha+1)} \\
\frac{L_{2} \delta_{2}\left(\beta t^{\beta-1}-t^{\beta}\right)}{\lambda_{2} \Gamma(\beta+1)} & \frac{L_{2} \delta_{2}\left(\beta t^{\beta-1}-t^{\beta}\right)}{\lambda_{2} \Gamma(\beta+1)}
\end{array}\right), \Phi_{m+1}= \\
& \left(\begin{array}{l}
\left\|x_{m+1}\left(t, x_{0}\right)-x_{m}\left(t, x_{0}\right)\right\| \\
\left\|y_{\mathrm{m}+1}\left(t, y_{0}\right)-y_{\mathrm{m}}\left(t, y_{0}\right)\right\|
\end{array}\right), \Phi_{m}=\left(\begin{array}{l}
\left\|x_{m}-x_{m-1}\right\| \\
\left\|y_{m}-y_{m-1}\right\|
\end{array}\right)
\end{aligned}
$$

Take the maximum value for both sides of (3.3):

$$
\Phi_{m+1} \leq \Delta_{0} \Phi_{m}
$$

where $\quad \Delta_{0}=\max _{t \in[0,1]} \Delta(t), \quad \Delta_{0}=\left(\begin{array}{ll}\frac{L_{1} \delta_{1}(\alpha-1)}{\lambda_{1} \Gamma(\alpha+1)} & \frac{L_{1} \delta_{1}(\alpha-1)}{\lambda_{1} \Gamma(\alpha+1)} \\ \frac{L_{2} \delta_{2}(\beta-1)}{\lambda_{2} \Gamma(\beta+1)} & \frac{L_{2} \delta_{2}(\beta-1)}{\lambda_{2} \Gamma(\beta+1)}\end{array}\right)$

By repletion of (3.4) we obtain:

$$
\Phi_{m+1} \leq \Lambda_{0}^{m} \Phi_{1}
$$

$\Phi_{1} \leq\left(\begin{array}{c}\frac{M_{1} \delta_{1}(\alpha-1)+\lambda_{1} b_{1} \alpha}{\lambda_{1} \Gamma(\alpha+1)} \\ \frac{M_{2} \delta_{2}(\beta-1)+\lambda_{2} b_{2} \beta}{\lambda_{2} \Gamma(\beta+1)}\end{array}\right)$ and also we get: $\sum_{i=1}^{m} \Phi_{i} \leq \sum_{i=1}^{m} \Delta_{0}^{i-1} \Phi_{1}$

Since the matrix $\Delta_{0}$ has eigenvalue $\lambda_{1}=0$,

$$
\lambda_{2}=\lambda_{\max }\left(\Delta_{0}\right)=\frac{M_{1} \delta_{1}(\alpha-1)+\lambda_{1} b_{1} \alpha}{\lambda_{1} \Gamma(\alpha+1)}+\frac{M_{2} \delta_{2}(\beta-1)+\lambda_{2} b_{2} \beta}{\lambda_{2} \Gamma(\beta+1)}<1
$$

the series (3.5) is uniformly convergent, i.e.

$$
\lim _{m \rightarrow \infty} \sum_{i=1}^{m} \Delta_{0}^{i-1} \Phi_{1}=\sum_{i=1}^{\infty} \Delta_{0}^{i-1} \Phi_{1}=\left(I-\Delta_{0}\right)^{-1} \Phi_{1}
$$

Thus the limiting relation (3.6) signifies uniform convergence of sequences:

$\left\{x_{m}\left(t, x_{0}\right)\right\}_{m=0}^{\infty},\left\{y_{m}\left(t, y_{0}\right)\right\}_{m=0}^{\infty}$, that is:

$$
\lim _{m \rightarrow \infty} x_{m}\left(t, x_{0}\right)=x\left(t, x_{0}\right), \text { and } \lim _{m \rightarrow \infty} y_{m}\left(t, y_{0}\right)=y\left(t, y_{0}\right)
$$

By all conditions and inequalities of the theorem the estimate

$$
\left(\begin{array}{l}
\left\|x_{m+1}\left(t, x_{0}\right)-x_{m}\left(t, x_{0}\right)\right\| \\
\left\|y_{\mathrm{m}+1}\left(t, y_{0}\right)-y_{\mathrm{m}}\left(t, y_{0}\right)\right\|
\end{array}\right) \leq \Delta_{0}^{m}\left(I-\Delta_{0}\right)^{-1} \Phi_{1}
$$

Is hold for all $\mathrm{m}=1,2, \ldots$

To prove that $x\left(t, x_{0}\right) \epsilon D_{1}$ and $y\left(t, y_{0}\right) \epsilon D_{2}$ we prove that:

$$
\begin{aligned}
\lim _{m \rightarrow \infty}\left(\frac{t^{\alpha-1}}{\Gamma(\alpha)} \int_{0}^{1}\right. & \int_{-\infty}^{t} K(t, s) F\left(t, s, x_{m}(s), y_{m}(s)\right) d s d s \\
& \left.-\int_{0}^{t} \frac{(t-s)^{\alpha-1}}{\Gamma(\alpha)}\left(\int_{-\infty}^{t} K(t, s) F\left(t, s, x_{m}(s), y_{m}(s)\right) d s\right) d s+\frac{b_{1} t^{\alpha-1}}{\Gamma(\alpha)}\right)=
\end{aligned}
$$




$$
\begin{aligned}
& \frac{t^{\alpha-1}}{\Gamma(\alpha)} \int_{0}^{1} \int_{-\infty}^{t} K(t, s) F(t, s, x(s), y(s)) d s d s- \\
& \int_{0}^{t} \frac{(t-s)^{\alpha-1}}{\Gamma(\alpha)}\left(\int_{-\infty}^{t} K(t, s) F(t, s, x(s), y(s)) d s\right) d s+\frac{b_{1} t^{\alpha-1}}{\Gamma(\alpha)} \\
& \lim _{m \rightarrow \infty}\left(\frac{t^{\beta-1}}{\Gamma(\beta)} \int_{0}^{1} \int_{-\infty}^{t} G(t, s) H\left(t, s, x_{m}(s), y_{m}(s)\right) d s d s\right. \\
& -\int_{0}^{t} \frac{(t-s)^{\beta-1}}{\Gamma(\beta)}\left(\int_{-\infty}^{t} G(t, s) H\left(t, s, x_{m}(s), y_{m}(s)\right) d s\right) d s+\frac{b_{2} t^{\beta-1}}{\Gamma(\beta)}= \\
& \frac{t^{\beta-1}}{\Gamma(\beta)} \int_{0}^{1} \int_{-\infty}^{t} G(t, s) H(t, s, x(s), y(s)) d s d s- \\
& \int_{0}^{t} \frac{(t-s)^{\beta-1}}{\Gamma(\beta)}\left(\int_{-\infty}^{t} G(t, s) H(t, s, x(s), y(s)) d s\right) d s+\frac{b_{2} t^{\beta-1}}{\Gamma(\beta)}
\end{aligned}
$$

We have:

$$
\begin{aligned}
& \| \frac{t^{\alpha-1}}{\Gamma(\alpha)} \int_{0}^{1} \int_{-\infty}^{t} K(t, s) F\left(t, s, x_{m}(s), y_{m}(s)\right) d s d s \\
& -\int_{0}^{t} \frac{(t-s)^{\alpha-1}}{\Gamma(\alpha)}\left(\int_{-\infty}^{t} K(t, s) F\left(t, s, x_{m}(s), y_{m}(s)\right) d s\right) d s+\frac{b_{1} t^{\alpha-1}}{\Gamma(\alpha)} \\
& -\frac{t^{\alpha-1}}{\Gamma(\alpha)} \int_{0}^{1} \int_{-\infty}^{t} K(t, s) F(t, s, x(s), y(s)) d s d s \\
& +\int_{0}^{t} \frac{(t-s)^{\alpha-1}}{\Gamma(\alpha)}\left(\int_{-\infty}^{t} K(t, s) F(t, s, x(s), y(s)) d s\right) d s-\frac{b_{1} t^{\alpha-1}}{\Gamma(\alpha)} \\
& \leq \frac{t^{\alpha-1}}{\Gamma(\alpha)} \int_{0}^{1} \int_{-\infty}^{t} \delta_{1} e^{-\lambda_{1}(t-s)}\left\|F\left(t, s, x_{m}(s), y_{m}(s)\right)-F(t, s, x(s), y(s))\right\| d s d s \\
& -\int_{0}^{t} \frac{(t-s)^{\alpha-1}}{\Gamma(\alpha)}\left(\int_{-\infty}^{t} \delta_{1} e^{-\lambda_{1}(t-s)} \| F\left(t, s, x_{m}(s), y_{m}(s)\right)\right. \\
& -F(t, s, x(s), y(s)) \| d s) d s \\
& \leq \frac{L_{1} \delta_{1}\left(\alpha t^{\alpha-1}-t^{\alpha}\right)}{\lambda_{1} \Gamma(\alpha+1)}\left(\left\|x_{m}-x\right\|+\left\|y_{m}-y\right\|\right)
\end{aligned}
$$

And for the function $\mathrm{y}\left(\mathrm{t}, y_{0}\right)$ we have

$$
\begin{aligned}
\| \frac{t^{\beta-1}}{\Gamma(\beta)} \int_{0}^{1} \int_{-\infty}^{t} G(t, s) H\left(t, s, x_{m}(s), y_{m}(s)\right) d s d s \\
\quad-\int_{0}^{t} \frac{(t-s)^{\beta-1}}{\Gamma(\beta)}\left(\int_{-\infty}^{t} G(t, s) H\left(t, s, x_{m}(s), y_{m}(s)\right) d s\right) d s+\frac{b_{2} t^{\beta-1}}{\Gamma(\beta)} \\
\quad-\frac{t^{\beta-1}}{\Gamma(\beta)} \int_{0}^{1} \int_{-\infty}^{t} G(t, s) H(t, s, x(s), y(s)) d s d s \\
+\int_{0}^{t} \frac{(t-s)^{\beta-1}}{\Gamma(\beta)}\left(\int_{-\infty}^{t} G(t, s) H(t, s, x(s), y(s)) d s\right) d s-\frac{b_{2} t^{\beta-1}}{\Gamma(\beta)} \|
\end{aligned}
$$




$$
\leq \frac{L_{2} \delta_{2}\left(\beta t^{\beta-1}-t^{\beta}\right)}{\lambda_{2} \Gamma(\beta+1)}\left(\left\|x_{m}-x\right\|+\left\|y_{m}-y\right\|\right)
$$

And since the sequences: $\left\{x_{m}\left(t, x_{0}\right)\right\}_{m=0}^{\infty},\left\{y_{m}\left(t, y_{0}\right)\right\}_{m=0}^{\infty}$ uniformly convergence to $x\left(t, x_{0}\right), y\left(t, y_{0}\right)$ respectively on the interval $[0,1]$, that is $(3.7),(3.8)$ satisfies.

Theorem (3.2): If all conditions and assumptions of theorem (3.1) satisfied, then the functions $x\left(t, x_{0}\right), y\left(t, y_{0}\right)$ are unique solution for system (1.1) on domain (1.2).

Proof: let

$$
\begin{gathered}
\left(\begin{array}{c}
u\left(t, u_{0}\right) \\
w\left(t, w_{0}\right)
\end{array}\right)= \\
\left(\begin{array}{l}
\frac{t^{\alpha-1}}{\Gamma(\alpha)} \int_{0}^{1} \int_{-\infty}^{t} K(t, s) F(t, s, u(s), w(s)) d s d s-\int_{0}^{t} \frac{(t-s)^{\alpha-1}}{\Gamma(\alpha)}\left(\int_{-\infty}^{t} K(t, s) F(t, s, u(s), w(s)) d s\right) d s+\frac{b_{1} t^{\alpha-1}}{\Gamma(\alpha)} \\
\frac{t^{\beta-1}}{\Gamma(\beta)} \int_{0}^{1} \int_{-\infty}^{t} G(t, s) H(t, s, u(s), w(s)) d s d s-\int_{0}^{t} \frac{(t-s)^{\beta-1}}{\Gamma(\beta)}\left(\int_{-\infty}^{t} G(t, s) H(t, s, u(s), w(s)) d s\right) d s+\frac{b_{2} t^{\beta-1}}{\Gamma(\beta)}
\end{array}\right)
\end{gathered}
$$

be another solution for system (1.1) then:

$$
\begin{gathered}
\left\|x\left(t, x_{0}\right)-u\left(t, u_{0}\right)\right\| \leq \frac{t^{\alpha-1}}{\Gamma(\alpha)} \int_{0}^{1} \int_{-\infty}^{t}\|K(t, s)\| \| F(t, s, x(s), y(s))- \\
F(t, s, u(s), w(s)) \| d s d s-\int_{0}^{t} \frac{(t-s)^{\alpha-1}}{\Gamma(\alpha)}\left(\int_{-\infty}^{t}\|K(t, s)\| \| F(t, s, x(s), y(s))-\right. \\
F(t, s, u(s), w(s)) \| d s) d s \\
\leq \frac{L_{1} \delta_{1}\left(\alpha t^{\alpha-1}-t^{\alpha}\right)}{\lambda_{1} \Gamma(\alpha+1)}(\|x-u\|+\|y-w\|)
\end{gathered}
$$

And

$$
\begin{aligned}
& \left\|y\left(t, y_{0}\right)-w\left(t, w_{0}\right)\right\| \leq \frac{t^{\alpha-1}}{\Gamma(\alpha)} \int_{0}^{1} \int_{-\infty}^{t}\|G(t, s)\| \| H(t, s, x(s), y(s))- \\
& H(t, s, u(s), w(s)) \| d s d s-\int_{0}^{t} \frac{(t-s)^{\alpha-1}}{\Gamma(\alpha)}\left(\int_{-\infty}^{t}\|G(t, s)\| \| H(t, s, x(s), y(s))-\right. \\
& H(t, s, u(s), w(s)) \| d s) d s
\end{aligned}
$$

Rewrite in vector form:

$$
\leq \frac{L_{2} \delta_{2}\left(\beta t^{\beta-1}-t^{\beta}\right)}{\lambda_{2} \Gamma(\beta+1)}(\|x-u\|+\|y-w\|)
$$

$$
\left(\begin{array}{l}
\left\|x\left(t, x_{0}\right)-u\left(t, u_{0}\right)\right\| \\
\left\|y\left(t, y_{0}\right)-\mathrm{w}\left(t, w_{0}\right)\right\|
\end{array}\right) \leq \Delta(t)\left(\begin{array}{l}
\|x(t)-u(t)\| \\
\|y(t)-w(t)\|
\end{array}\right)
$$

By take the maximum value for both sides of (3.9) and reputation it we get:

$$
\left(\begin{array}{l}
\left\|x\left(t, x_{0}\right)-u\left(t, u_{0}\right)\right\| \\
\left\|y\left(t, y_{0}\right)-\mathrm{w}\left(t, w_{0}\right)\right\|
\end{array}\right) \leq \Delta_{0}^{m}\left(\begin{array}{l}
\|x(t)-u(t)\| \\
\|y(t)-u(t)\|
\end{array}\right)
$$

From (3.9) and condition (1.8) we have $A_{0}^{m} \rightarrow 0$ when $m \rightarrow \infty$ that is:

$$
x\left(t, x_{0}\right)=u\left(t, u_{0}\right) \text { and } y\left(t, y_{0}\right)-\mathrm{w}\left(t, w_{0}\right)
$$

Therefor $x\left(t, x_{0}\right), y\left(t, y_{0}\right)$ is a unique solution for system (1.1). 
Theorem (3.3): Under the hypothesis and conditions of theorem (3.1) if $\tilde{x}\left(t, x_{0}\right), \tilde{y}\left(t, y_{0}\right)$ is any other solution of system (1.1), then the solution is stable if satisfies the inequality:

Where:

$$
\left(\begin{array}{l}
\left\|x\left(t, x_{0}\right)-\tilde{x}\left(t, x_{0}\right)\right\| \\
\left\|y\left(t, y_{0}\right)-\tilde{y}\left(t, y_{0}\right)\right\|
\end{array}\right) \leq\left(\begin{array}{l}
\epsilon_{1} \\
\epsilon_{2}
\end{array}\right)
$$

$$
\begin{aligned}
& \tilde{x}\left(t, x_{0}\right)=\frac{t^{\alpha-1}}{\Gamma(\alpha)} \int_{0}^{1} \int_{-\infty}^{t} K(t, s) F(t, s, \tilde{x}(s), \tilde{y}(s)) d s d s- \\
& \int_{0}^{t} \frac{(t-s)^{\alpha-1}}{\Gamma(\alpha)}\left(\int_{-\infty}^{t} K(t, s) F(t, s, \tilde{x}(s), \tilde{y}(s)) d s\right) d s+ \\
& \frac{b_{1} t^{\alpha-1}}{\Gamma(\alpha)} \\
& \tilde{y}\left(t, y_{0}\right)=\frac{t^{\beta-1}}{\Gamma(\beta)} \int_{0}^{1} \int_{-\infty}^{t} G(t, s) H(t, s, \tilde{x}(s), \tilde{y}(s)) d s d s- \\
& \int_{0}^{t} \frac{(t-s)^{\beta-1}}{\Gamma(\beta)}\left(\int_{-\infty}^{t} G(t, s) H(t, s, \tilde{x}(s), \tilde{y}(s)) d s\right) d s+ \\
& \left.\begin{array}{c}
\left\|x\left(t, x_{0}\right)-\tilde{x}\left(t, x_{0}\right)\right\| \leq \frac{L_{1} \delta_{1}\left(\alpha t^{\alpha-1}-t^{\alpha}\right)}{\lambda_{1} \Gamma(\alpha+1)}(\|x-\tilde{x}\|+\|y-\tilde{y}\|) \\
\left\|y\left(t, y_{0}\right)-\tilde{y}\left(t, y_{0}\right)\right\| \leq \frac{L_{2} \delta_{2}\left(\beta t^{\beta-1}-t^{\beta}\right)}{\lambda_{2} \Gamma(\beta+1)}(\|x-\tilde{x}\|+\|y-\tilde{y}\|)
\end{array}\right\}
\end{aligned}
$$

Rewrite (3.11), (3.12) in victor form we get:

$$
\left(\begin{array}{l}
\left\|x\left(t, x_{0}\right)-\tilde{x}\left(t, x_{0}\right)\right\| \\
\left\|y\left(t, y_{0}\right)-\tilde{y}\left(t, y_{0}\right)\right\|
\end{array}\right) \leq \Delta(t)\left(\begin{array}{l}
\|x(t)-\bar{x}(t)\| \\
\|y(t)-\bar{y}(t)\|
\end{array}\right)
$$

By condition (1.8) and for $\epsilon_{1}, \epsilon_{2} \geq 0$ we have:

$$
\left(\begin{array}{l}
\left\|x\left(t, x_{0}\right)-\tilde{x}\left(t, x_{0}\right)\right\| \\
\left\|y\left(t, y_{0}\right)-\tilde{y}\left(t, y_{0}\right)\right\|
\end{array}\right) \leq\left(\begin{array}{l}
\epsilon_{1} \\
\epsilon_{2}
\end{array}\right)
$$

By the definition of stability, we find that $\tilde{x}\left(t, x_{0}\right), \tilde{y}\left(t, y_{0}\right)$ is stable solution for system (1.1)

Example (3.1): consider the following system of fractional integrodifferential equations:

$$
\left.\begin{array}{l}
D^{4 / 3} x(t)=\int_{-\infty}^{t}\left(4 e^{2 s}+1\right) \frac{x(s)}{y(s)} d s \\
D^{3 / 2} y(t)=\int_{-\infty}^{t} 3 \cos (2 s)(y(s)+\sin (x(s))) d s \\
D^{1 / 3} x(0)=0, \quad D^{1 / 3} x(1)=2, \quad D^{1 / 2} x(0)=0, \quad D^{1 / 2} x(1)=3
\end{array}\right]
$$

Comparing (3.14) and (1.1) we see that, $\alpha=4 / 3, \beta=3 / 2, k(t, s)=\left(4 e^{2 s}+\right.$ 1), $G(t, s)=3 \cos (2 s)$ $F(t, s, x(s), y(s))=x(s) / y(s), H(t, s, x(s), y(s))=y(s)+\sin (x(s))$, if we choose $M_{1}=1$, $M_{2}=1, L_{1}=2 M_{1}, L_{2}=2 M_{2}, \delta_{1}=1, \delta_{2}=2, \lambda_{1}=2, \lambda_{2}=3$, then (1.3) - (1.6) holds and : 


$$
\lambda_{\max }\left(\Delta_{0}\right)=\frac{L_{1} \delta_{1}(\alpha-1)}{\lambda_{1} \Gamma(\alpha+1)}+\frac{L_{2} \delta_{2}(\beta-1)}{\lambda_{2} \Gamma(\beta+1)}=\frac{(2)(1)(4 / 3-1)}{(2)(1.1906)}+\frac{(2)(2)(3 / 2-1)}{(3)(1.3293)}<1
$$

Thus, by Theorems (3.1) -(3.3), we obtain that (1.1) has a unique stability solution.

\section{Conclusions}

The article presented some existence and uniqueness results for a boundary value problem of fractional integro-differential system. The prove of the theorems based on two basic conditions (1.8), (3.1). The basic of fractional differentiation were used to find the solution formula. The idea of existence and uniqueness theorem is the basis for finding results. The present work can be extended to boundary value problem with nonlocal and nonseparated fractional boundary conditions.

\section{References}

[1] Podlubny, I: Fractional differential equations, mathematics in science and engineering. Academic Press, New York/London/Toronto (1999)

[2] R. Hilfer, Applications of Fractional Calculus in Physics. World Scientific,Singapore (2000).

[3] V. Kac and P. Cheung, Quantum Calculus, Springer, New York, NY, USA, 2002.

[4] V. Tarasov, Fractional Dynamics: Applications of Fractional Calculus to Dynamics of Particles, Fields and Media. Springer-Verlag, New York (2011).

[5] M. H. Annaby and Z. S. Mansour, q-Fractional Calculus and Equations, Springer, Berlin, Germany, 2012.

[6] W. A. Al-Salam, "Some fractional q-integrals and q-derivatives,"Proceedings of the Edinburgh Mathematical Society, vol. 15, pp.135-140, 1966-1967.

[7] R. P. Agarwal, "Certain fractional q-integrals and q-derivatives,"Proceedings of the Cambridge Philosophical Society, vol. 66, pp.365-370, 1969.

[8] P. M. Rajkovi'c, S. D. Marinkovi'c, and M. S. Stankovi'c, "Fractional integrals and derivatives in q-calculus," Applicable Analysis and Discrete Mathematics, vol. 1, no. 1, pp. 311-323, 2007.

[9] M. H. Annaby and Z. S.Mansour, "q-Taylor and interpolation series for Jackson qdifference operators," Journal of Mathematical Analysis and Applications, vol. 344, no. 1, pp. 472-483, 2008

[10] J. Henderson and R. Luca, Positive solutions for a system of nonlocal fractional boundary value problems. Fract. Calc. Appl. Anal. 16, No 4 (2012), 985-1008; DOI: 10.2478/s13540-013-0061-4.

[11] J. R. Graef, L. Kong, Q. Kong, and M. Wang, Uniqueness of positive solutions of fractional boundary value problems withnon-homogeneous integral boundary conditions. Fract. Calc. Appl. Anal. 15, No 3 (2012).

[12] J. R. Graef, L. Kong, and B. Yang, Positive solutions for a semi posit one fractional boundary value problem with a forcing term. Fract. Calc.Appl. Anal. 15, No 1 (2012), 8-24; $\quad$ DOI: $10.2478 / \quad$ s13540-012-0002-7; http://link.springer.com/article/10.2478/s13540-012-0002-7.

[13] J. R. Graef, L. Kong, Q. Kong, and M. Wang, Fractional bound a ray value problem with integral boundary conditions. Appl. Anal. 92(2013).

[14] J. R. Graef and L. Kong, Existence of positive solutions to a higher order singular boundary value problem with fractional q-derivatives. Fract. Calc. Appl. Anal. 16, No 3 (2013), 695-708; DOI: 10.2478/s13540-013-0044-5; http://link.springer.com/article/10.2478/s13540-013-0044-5.

[15] J. R. Graef, L. Kong, Q. Kong, and M. Wang, Positive solutions of nonlocal fractional boundary value problems. Discrete Contin. Dyn. Syst., Suppl. 2013. 
[16] J. R. Graef, L. Kong, Q. Kong, and M. Wang, Existence and uniqueness of solutions for a fractional boundary value problem with Dirichlet boundary condition. Electron. J. Qual. Theory Differ. Equ. 2013.

[17] N. Nyamoradi and H. Alaei Dizaji "Existence solutions for nonlocal fractional differential equation with nonlinear boundary conditions", IJST (2014) 38A4: 455461 Iranian Journal of Science \& Technology.

[18] N. Nyamoradi and H. Alaei Dizaji, "Existence solutions for nonlocal fractional differential equation with nonlinear boundary conditions", Iranian Journal of Science \& Technology IJST (2014) 38A4: 455-461.

[19] Zhenyu GUO, Min LIU, and Zhijing WANG, "Existence and Uniqueness of Solutions for a Nonlinear Fractional Integrodifferential Equation with Three-Point Fractional Boundary Conditions", Journal of Mathematical Research with Applications, Vol. 36, No. 1, pp. 79-86, Jan 2016. DOI: 10.3770/j.issn:20952651.2016.01.010.

[20] Butris and Ishak, "Some result's in the theory of fractional integral equations of Volterra-Fredholm types “, Volume 9, No.1, January - February 2020 International Journal of Information Systems and Computer Sciences https://doi.org/10.30534/ijiscs/2020. 